\title{
Immunological evaluation of invasive Lactobacillus plantarum co-expressing EtMIC2 and chicken interleukin-18 against Eimeria tenella
}

\author{
Zan Zhang ${ }^{1} \cdot$ Hai-Bin Huang ${ }^{1} \cdot$ Yan-Long Jiang ${ }^{1} \cdot$ Jing Liu ${ }^{1} \cdot$ Xing Gao ${ }^{1} \cdot$ Yang Liu ${ }^{1}$ • Wen-Tao Yang ${ }^{1} \cdot$ Chun-Wei Shi $^{1}$ • \\ Dan Wang ${ }^{1} \cdot$ Jian-Zhong Wang ${ }^{1} \cdot$ Yuan-Huan Kang ${ }^{1} \cdot$ Chun-Feng Wang ${ }^{1} \cdot$ Gui-Lian Yang ${ }^{1}$ (i)
}

Received: 29 December 2018 / Accepted: 1 June 2020 / Published online: 27 July 2020

(C) Springer-Verlag GmbH Germany, part of Springer Nature 2020

\begin{abstract}
Chicken coccidiosis is a protozoan parasitic disease that leads to considerable economic losses in the poultry industry. In this study, we used invasive Lactobacillus plantarum (L.P) expressing the FnBPA protein as a novel bacterial carrier for DNA delivery into epithelial cells to develop a live oral DNA vaccine. A fusion DNA vaccine co-expressing EtMIC2 and chicken IL18 (chIL-18) was constructed and then delivered to the host by invasive L.P. Its efficacy against Eimeria tenella challenge was evaluated in chickens by examining the relative weight gain rate; caecal lesion score; OPG; anti-coccidial index (ACI); levels of EtMIC2 antibody, FnBPA, IL-4, IL-18, IFN- $\gamma$ and SIgA; and proliferation ability and percentages of $\mathrm{CD} 4^{+}$and $\mathrm{CD} 8^{+}$ splenocytes. The experimental results showed that chickens immunized with invasive L.P carrying the eukaryotic expression vector pValac-EtMIC2 (pValac-EtMIC2/pSIP409-FnBPA) had markedly improved immune protection against challenge compared with that of chickens immunized with non-invasive L.P (pValac-EtMIC2/pSIP409). However, invasive L.P co-expressing EtMIC2 with the chIL-18 vector exhibited the highest protection efficiency against $E$. tenella. These results indicate that invasive Lactobacillus-expressing FnBPA improved humoural and cellular immunity and enhanced resistance to E. tenella. The DNA vaccine delivered by invasive Lactobacillus provides a new concept and method for the prevention of $E$. tenella.
\end{abstract}

Keywords Coccidiosis $\cdot$ Invasive Lactobacillus plantarum $\cdot$ EtMIC2 $\cdot$ chIL-18 $\cdot$ DNA delivery

\section{Introduction}

Eimeria tenella is a parasitic protozoan and the most pathogenic of the seven species of coccidia that infect chickens, causing significant economic losses in the avian industry worldwide. The primary control measures for coccidiosis are the widespread use of anti-coccidial drugs and live oocyst vaccines (Shirley et al.

Section Editor: Xing-Quan ZHU

Chun-Feng Wang

wangchunfeng@jlau.edu.cn

Gui-Lian Yang

yangguilian@jlau.edu.cn

1 College of Animal Science and Technology, Jilin Provincial Engineering Research Center of Animal Probiotics, Key Laboratory of Animal Production and Product Quality Safety of Ministry of Education, Jilin Agricultural University, 2888 Xincheng Street, Changchun 130118, China
2005). Nevertheless, anti-coccidial drugs have long been used to prevent coccidial infections, resulting in the emergence of drugresistant Eimeria strains (Liu et al. 2011). Moreover, the live oocyst vaccine has a number of disadvantages, including reversibility of coccidial virulence, pathogenicity and high production costs (Vermeulen 1998; Sharman et al. 2010).

Immunization against chicken coccidiosis is greatly dependent on cellular immunity (Rose and Hesketh 1982). The strategy of using live bacterial cells as vehicles to deliver recombinant eukaryotic vectors that carry antigens has emerged over the past two decades as an interesting alternative for the development of new vaccines. Lactobacillus plantarum $(L . P)$ is considered a generally recognized as safe (GRAS), non-invasive lactic acid bacterium (LAB); LABs are probiotics that have immunomodulatory functions and are widely used in health care and industrial fermentation (Isolauri et al. 2001; Karczewski et al. 2010; Kotzamanidis et al. 2010; Gunal et al. 2006). L.P can be used as an improved vector to carry plasmids expressing foreign proteins into hosts 
and to induce an intestinal mucosal immune response against coccidiosis if its adhesion and invasiveness in the intestine are enhanced.

Fibronectin-binding protein A (FnBPA) produced by Staphylococcus aureus is an invasive protein that mediates adhesion (Innocentin et al. 2009). Its expression on the surface of recombinant LABs can improve the efficiency of plasmid delivery (Almeida et al. 2014). In our laboratory, FnBPA was ligated into the pSIP409 vector to generate recombinant L.P capable of expressing the FnBPA protein with an invasive function. On this basis, we transformed the eukaryotic plasmid pValac, which is a new plasmid vector for DNA delivery, to carry the antigen gene to form a microecological preparation containing a double expression plasmid.

The E. tenella microneme-2 (EtMIC2) protein is secreted from the microneme and plays an important role in the early stage of host cell invasion during infection with coccidia (Tomley and Soldati 2001). Several studies have confirmed that the recombinant EtMIC2 protein shows good immunogenicity and might be a good candidate for use in vaccine development (Ding et al. 2005; Sathish et al. 2011; Zhang et al. 2014). To enhance its anti-coccidial effect, several cytokines were inserted into eukaryotic expression plasmids in tandem with antigen genes to immunize the host (Geriletu et al. 2011; Song et al. 2013; Song et al. 2015; Song et al. 2016). In recent years, studies have revealed that the cytokine chIL-18 could enhance the protective efficacy of immunization and host immune responses (Shi et al. 2014). IL-18 can induce Th2mediated humoural immunity and Th1-mediated cellular immunity, enhance the activity of CTLs and NK cells and induce FasL-mediated cytotoxicity in immune cells to achieve a defence response (Wong et al. 2013; Kinoshita et al. 2013). In this study, a fusion DNA vaccine co-expressing EtMIC2 and chIL-18 was constructed and then carried into the host by invasive L.P to prevent coccidiosis.

\section{Materials and methods}

\section{Plasmids, bacterial strains, parasites and animals}

The plasmids and bacterial strains used in this work are listed in Table 1. L.P NC8 cells were grown in MRS medium at $30{ }^{\circ} \mathrm{C}$ without shaking. Escherichia coli (E. coli) TG1 cells were grown in Luria-Bertani (LB) medium at $37{ }^{\circ} \mathrm{C}$ with vigorous shaking. Solid media were prepared by adding $1.5 \%(\mathrm{w} / \mathrm{v})$ agar to broth. Antibiotics were added at the following concentrations as necessary: $200 \mu \mathrm{g} / \mathrm{mL}$ erythromycin for E. coli, $10 \mu \mathrm{g} / \mathrm{mL}$ erythromycin for $L . P$ and $10 \mu \mathrm{g} / \mathrm{mL}$ chloramphenicol. HEK-293T cells were preserved at Jilin Animal Ecological Engineering Research Center. The wildtype E. tenella strain was stored in our laboratory. Sporulated oocysts were stored in $2.5 \%$ potassium dichromate solution at
$4{ }^{\circ} \mathrm{C}$. Newly hatched broiler chickens were raised in a sterile space under coccidia-free conditions until the end of the experiment. Food and water without anti-coccidia drugs were available ad libitum. All animal husbandry and experimental procedures were performed in accordance with the Chinese Animal Management Ordinance (People's Republic of China Ministry of Health, document No. 55, 2001). The protocol for the animal studies was approved by the Animal Care and Ethics Committees of Jilin Agriculture University.

\section{Construction of the recombinant plasmids pValac- EtMIC2 and pValac-EtMIC2-IL18}

The sequences of EtMIC2 (FJ807654.1) and IL-18 (AY775781.1) were obtained from GenBank. pUC57EtMIC2-IL18 was optimized and routinely synthesized by GENEWIZ (Suzhou, China). The plasmid pValac was used as a new eukaryotic expression vector to generate the recombinant plasmids. The entire coding region of the EtMIC2 gene was amplified by PCR from the pUC57-EtMIC2-IL18 plasmid using the primers EtMIC2-F (5'-ATAGGTACC ATGGTGATGCAGCTGACG-3') and EtMIC2-R (5'TGGAAGCTTCTAGAGCACGGGCTTTGTC-3'). The products of PCR amplification were digested with Kpn I and $X b a$ I and purified by a DNA Clean-up Kit (CW2301) from CoWin Biosciences. Then, the purified products of the EtMIC2 gene were ligated into pValac, which was digested with the same enzymes that were used to digest the PCR products, to generate the plasmid pValac-EtMIC2 (Fig. 1a). The EtMIC2-IL18 gene was amplified by PCR using the primers EtMIC2-F and IL18-R (5'-ATA TCTAGATC AGTGGTGGTGGTGGTGG-3'). The pValac-EtMIC2-IL18 plasmid was constructed in the same manner (Fig. 1b). The results of PCR amplification, double enzyme digestion and sequencing confirmed successful plasmid construction.

\section{Expression analysis of the recombinant plasmids in vitro}

Western blotting and indirect immunofluorescence analysis (IFA) were used to detect whether the recombinant plasmid expressed the antigen proteins.

A series of plasmids, pValac-gfp, pValac-EtMIC2 and pValac-EtMIC2-IL18, were transferred to HEK-293T cells with Lipofectamine 3000 reagent (Invitrogen, USA) according to the manufacturer's instructions and cultured in 6- and 48-well plates. Forty-eight hours after transfection, the cells were gently washed with PBS three times, the pre-cooled extraction reagent was added to the 6-well plate and the cells were fully lysed. The lysate was transferred to a centrifuge tube, and the cells were fully lysed in an ice bath for $20 \mathrm{~min}$ and centrifuged at $12,000 \mathrm{rpm}$ for $5 \mathrm{~min}$ at $4{ }^{\circ} \mathrm{C}$. The cell proteins in the supernatant were separated by $8 \%$ SDS- 
Table 1 Bacterial strains, plasmids and primers used in this work

\begin{tabular}{lll}
\hline Strains & Characteristics and plasmids & Reference \\
\hline Non-invasive L. plantarum NC8 & Em, pSIP409 & Lab source \\
Invasive L. plantarum NC8 & Em, pSIP409-FnBPA & Lab source \\
E. coli TG1 & Cm, pValac & TaKaRa Corporation, Japan \\
pValac/pSIP409 & Em, Cm, double plasmids & This work \\
pValac-EtMIC2/pSIP409 & Em, Cm, double plasmids & This work \\
pValac-EtMIC2/pSIP409-FnBPA & Em, Cm, double plasmids & This work \\
pValac-EtMIC2-IL18/pSIP409 & Em, Cm, double plasmids & This work \\
pValac-EtMIC2-IL18/pSIP409-FnBPA & Em, Cm, double plasmids & This work \\
\hline
\end{tabular}

PAGE and then transferred to a polyvinylidene fluoride (PVDF) membrane (Millipore, USA) at $90 \mathrm{~mA}$ for $2 \mathrm{~h}$. Then, the PVDF membrane was blocked with 5\% skim milk powder overnight and incubated with anti-Flag Tag (1:1000) and anti-His Tag (1:1000) monoclonal antibodies, excluding the pValac-gfp group (negative control), at room temperature for $1 \mathrm{~h}$. After washing three times with TBST, the PVDF membranes were incubated with HRP-labelled goat antimouse IgG (1:500) for $1 \mathrm{~h}$. After washing the membranes with TBST, ECL substrates (Thermo Scientific Pierce) were used for chemiluminescence development.

Cells in 48-well plates were washed three times with PBS and incubated with anti-Flag tag $(1: 1000)$ and anti-His tag (1:1000) monoclonal antibodies at $37{ }^{\circ} \mathrm{C}$ for $1 \mathrm{~h}$, excluding the pValac-gfp group (positive control). Then, the plates were washed three times with PBST and incubated with FITClabelled goat anti-mouse $\operatorname{IgG}(1: 500)$ at $37{ }^{\circ} \mathrm{C}$ for $1 \mathrm{~h}$. After washing the plates in the same manner, the cells were
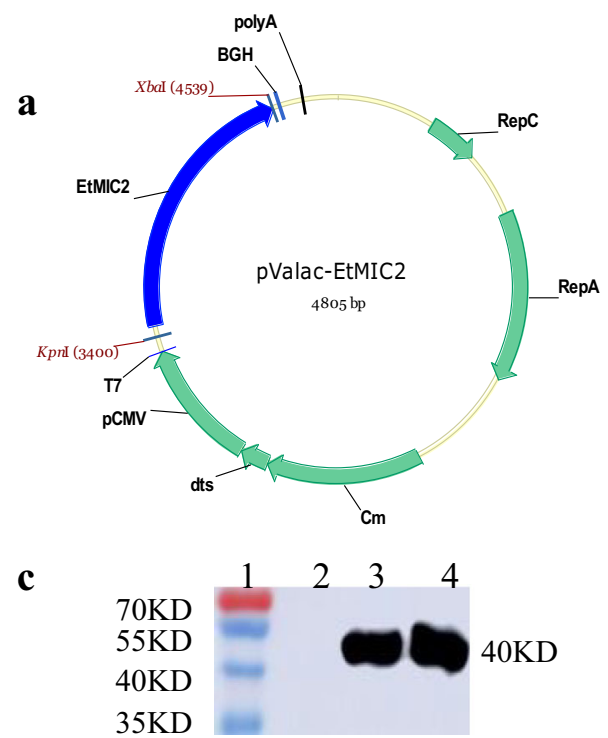

Fig. 1 Vectors and Western blot. The genes encoding EtMIC2 and EtMIC2-IL18 were inserted into the vector pValac, yielding pValacEtMIC2 (a) and pValac-EtMIC2-IL18 (b), respectively. The expression of EtMIC2 and EtMIC2-IL18 was confirmed by Western blotting using anti-Flag tag (c) and anti-His tag monoclonal antibodies (d) as primary incubated with dihydrochloride (DAPI) (1:2000). Finally, fluorescence was detected via confocal microscopy.

\section{Construction of invasive L.P NC8 harbouring plasmids}

The recombinant plasmids were added to invasive or noninvasive L.P NC8 competent cells, gently mixed and then transferred to a pre-cooled $0.2-\mathrm{cm}$ electrocuvette. After resting in an ice bath for $5 \mathrm{~min}$, the plasmids were placed into an electric transformation instrument $(2.5 \mathrm{kV}, 6 \mathrm{~ms})$ for electroporation, producing non-invasive NC8 (pValac-EtMIC2/ pSIP409 and pValac-EtMIC2-IL18/pSIP409) and invasive NC8 (pValac-EtMIC2/pSIP409-FnBPA and pValacEtMIC2-IL18/pSIP409-FnBPA). The four strains were then inoculated into MRS liquid culture medium at a ratio of 1:100 and cultured anaerobically at $37{ }^{\circ} \mathrm{C}$ for $24 \mathrm{~h}$. The $\mathrm{OD}_{600 \mathrm{~nm}}$ value was measured every $2 \mathrm{~h}$, and the growth curve of each strain was obtained.

b

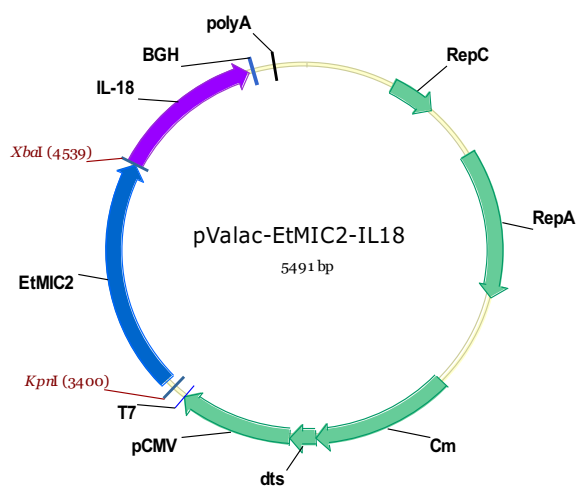

d

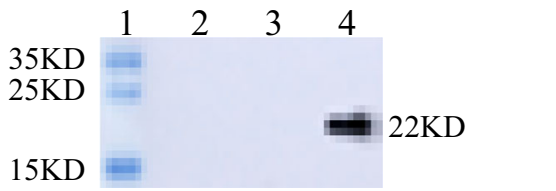

antibodies and HRP-conjugated goat anti-mouse $\operatorname{IgG}$ as the secondary antibody. Lane 1, protein marker; Lane 2, pValac without EtMIC2 and IL18 production; Lane 3 and Lane 4, EtMIC2 and IL-18 proteins expressed in $293 \mathrm{~T}$ cells transfected with pValac-EtMIC2 and pValacEtMIC2-IL18, respectively 


\section{Assays of bacterial invasion into CEF cells}

Nine-day-old SPF chicken embryos were selected, and the head, neck and internal organs were removed. The embryos were then cut into pieces and washed three times with PBS until the tissues were whitish, and $5 \%$ trypsin digestion was performed at $37{ }^{\circ} \mathrm{C}$ for $10 \mathrm{~min}$ in a $5 \% \mathrm{CO}_{2}$ atmosphere. The digestion was stopped with DMEM, and the samples were allowed to stand for approximately $10 \mathrm{~min}$. The supernatant was then discarded, and the cell pellet was resuspended to generate a homogeneous solution. The cells were filtered, and the filtrate was placed at $37^{\circ} \mathrm{C}$ for $24 \mathrm{~h}$ in a $5 \% \mathrm{CO}_{2}$ atmosphere.

The experimental strains carrying plasmids were inoculated into MRS (Em) medium at a ratio of 1:100 and cultured at $30{ }^{\circ} \mathrm{C}$ until $\mathrm{OD}_{600}=0.3$. The inducer pheromone SppIP $(50 \mathrm{ng} / \mathrm{mL})$ was then added, and the bacteria were incubated anaerobically until $\mathrm{OD}=1$. The four strains constructed above were added to CEF cells in a 24-well plate at a final multiplicity of infection (MOI) of approximately $10^{3}$ (bacteria/cell). After $3 \mathrm{~h}$ of stimulation, the cells were treated for $2 \mathrm{~h}$ with gentamicin $(20 \mathrm{mg} / \mathrm{ml})$ to kill extracellular bacteria. After multiple washes, the cells were plated on solid MRS (Em, $\mathrm{Cm}$ ), and the number of colonies was counted at $24 \mathrm{~h}$.

\section{Immunization and $E$. tenella challenge}

Chickens were divided into 6 groups randomly, with 20 birds in each group. At 1, 3, 5, 12, 14 and 16 days of age, the chickens in each group were orally immunized with the experimental strains and challenged according to the experimental design, as described in Table 2. All chickens except those in the unchallenged group (group I) were challenged orally with $5.0 \times 10^{4}$ sporulated oocysts of $E$. tenella at 30 days. The animals were euthanized 8 days post-challenge.

\section{Evaluation of immune protection}

Blood samples were collected from five chickens in each group on days 30 and 38 , and the serum was collected from the blood by centrifugation and stored at $-80{ }^{\circ} \mathrm{C}$ until use. The levels of the specific anti-EtMIC2 antibody and SIgA, IL-4, IL-18 and IFN- $\gamma$ were examined by enzyme-linked immunosorbent assay
(ELISA). Single-cell suspensions were prepared from spleens and peripheral blood leukocytes (PBLs) as described previously (Helmby et al. 2000; Nolte et al. 2015). The proliferation ability of cells in the PBLs was evaluated, and the percentages of $\mathrm{CD}^{+}$ and $\mathrm{CD} 8^{+}$splenocytes were evaluated by fluorescence-activated cell sorting (FACS).

After challenge with $E$. tenella, the body weight was recorded every day, and changes were observed. Faecal samples were randomly collected on the 6th, 7th and 8th days according to the fivepoint method, and 2-g samples from each group were stirred in a clean beaker containing $58 \mathrm{~mL}$ of saturated salt water. Then, $10 \mu \mathrm{L}$ of the suspension was extracted and added to a counter chamber under the coverslip, which was observed and counted under the microscope after several minutes. Finally, on the 8th day, the chickens were dissected, and the caecum was collected for observation. Then, the protective efficacy of each experimental group was evaluated on the basis of the body weight gain, relative growth rate, decrease in the oocyst shedding ratio, caecal lesion score and anti-coccidial index (ACI). The caecal lesion score of the chickens from each group was recorded on a numerical scale from 0 (normal) to 4 (severe) according the previously described method of Johnson and Reid (Johnson et al. 1970). To visualize the degree of damage in caecum lesions, the caecum tissues were fixed, dehydrated, cleared, waxed, embedded and dried, pathological sections were made, and H\&E staining was conducted to observe the pathological results under the microscope.

\section{Statistical analysis}

The data were statistically analysed using GraphPad Prism 5.0 (GraphPad Software). A paired $t$ test was used for differential analysis. $P<0.05$ was considered statistically significant. All data are expressed as the mean $\pm \mathrm{SEM}$.

\section{Results}

\section{Construction and expression analysis of recombinant plasmids}

The recombinant plasmids pValac-EtMIC2 and pValacEtMIC2-IL18 were constructed as described in 'Materials

Table 2 Experimental groups of chickens in immunization and challenge experiment

\begin{tabular}{llll}
\hline Groups & Strains & Immunization (dose) & Challenge $($ dose $)$ \\
\hline I & $/$ & Normal saline $/ 0.2 \mathrm{~mL}$ & $/$ \\
II & $/$ & Normal saline $/ 0.2 \mathrm{~mL}$ & $5.0 \times 10^{4} / 0.5 \mathrm{~mL}$ \\
III & pValac/pSIP409 & $1.0 \times 10^{9} \mathrm{CFU} / 0.2 \mathrm{~mL}$ & $5.0 \times 10^{4} / 0.5 \mathrm{~mL}$ \\
IV & pValac-EtMIC2 $/ \mathrm{pSIP} 409$ & $1.0 \times 10^{9} \mathrm{CFU} / 0.2 \mathrm{~mL}$ & $5.0 \times 10^{4} / 0.5 \mathrm{~mL}$ \\
V & pValac-EtMIC2 $/ \mathrm{pSIP} 409-F n B P A$ & $1.0 \times 10^{9} \mathrm{CFU} / 0.2 \mathrm{~mL}$ & $5.0 \times 10^{4} / 0.5 \mathrm{~mL}$ \\
VI & pValac-EtMIC2-IL18/pSIP409-FnBPA & $1.0 \times 10^{9} \mathrm{CFU} / 0.2 \mathrm{~mL}$ & $5.0 \times 10^{4} / 0.5 \mathrm{~mL}$ \\
\hline
\end{tabular}


and methods' (Fig. $1 \mathrm{a}$ and b). The Western blot results showed that $293 \mathrm{~T}$ cells transfected with the recombinant plasmid expressed the EtMIC2 and chIL-18 proteins with sizes of $40 \mathrm{kD}$ and $22 \mathrm{kD}$, respectively, which were very close to the anticipated sizes (Fig. $1 \mathrm{c}$ and d). The IFA results also confirmed that the proteins were expressed in 293T cells (Fig. 2).

\section{Calculation of the invasion rate of the bacterial strains}

The recombinant plasmids were serially electroporated into L.P carrying the empty vector pSIP409 without the FnBPA gene as the control group and the pSIP409 vector containing the FnBPA gene (as 'Construction of invasive L.P NC8 harbouring plasmids'), and the four strains were finally constructed. The growth curve is shown in Fig. 3a. By stimulating the CEFs isolated from chicken embryos and counting the number of intracellular bacteria, the cell invasion rates were calculated. The results indicated that the strains expressing the FnBPA protein on the surface were able to enter the cells more frequently and had a higher invasion rate than the cells in control group, and the difference was significant $(P<0.001)$ (Fig. 3b). Thus, FnBPA plays a crucial role in invasion.

\section{Detection of antibody levels by ELISA}

Both serum antibody and mucosal SIgA were detected to evaluate the immune response to stimulation by the experimental strains. The specific anti-EtMIC2 antibody level was detected in serum from chickens in all the groups, and the results indicated that the levels in the immunized chickens (groups IV, V and VI) were markedly higher than those in the controls (groups I, II and III) (Fig. 4a). The results showed that recombinant L.P carrying antigens could induce the host to produce more IFN- $\gamma$, and the content of IFN- $\gamma$ in group VI was the highest,
Fig. 2 Indirect

immunofluorescence. IFA detection of the EtMIC2 and IL-

18 proteins expressed in $293 \mathrm{~T}$ cells transfected with recombinant plasmids. a pValac without EtMIC2- and IL18-producing transfected cells was used as the negative control. b pValac with EGFP-producing transfected cells as the positive control. c pValacEtMIC2-transfected cells were stained with DAPI, an anti-Flag tag monoclonal antibody and FITC-conjugated goat anti-mouse IgG. d p Valac-EtMIC2-IL18transfected cells were stained with DAPI, an anti-Flag tag monoclonal antibody and FITC-

conjugated goat anti-mouse IgG. e pValac-EtMIC2-IL18-

transfected cells were stained with DAPI, an anti-His tag monoclonal antibody and FITC-conjugated goat anti-mouse IgG. Arrows indicate the expression of the target protein in $293 \mathrm{~T}$ cells
DAPI

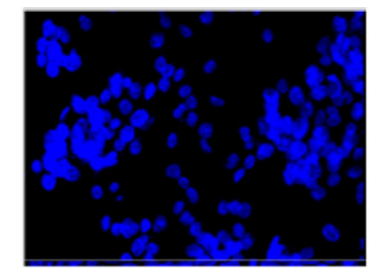

b

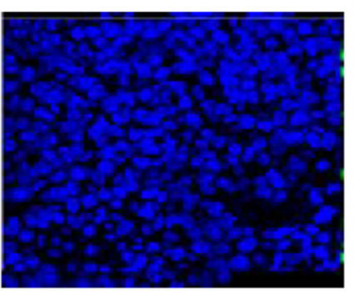

c

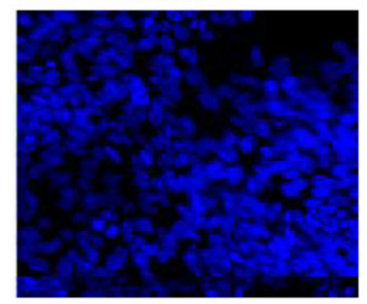

d

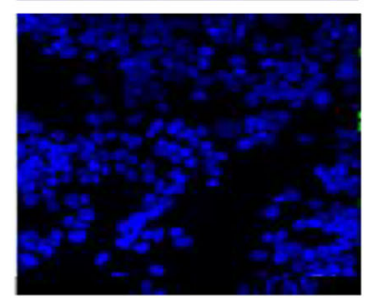

e

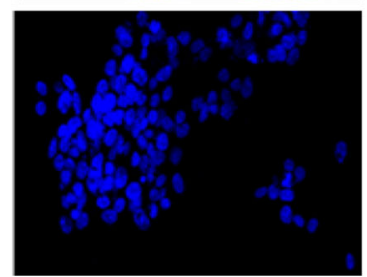

FITC
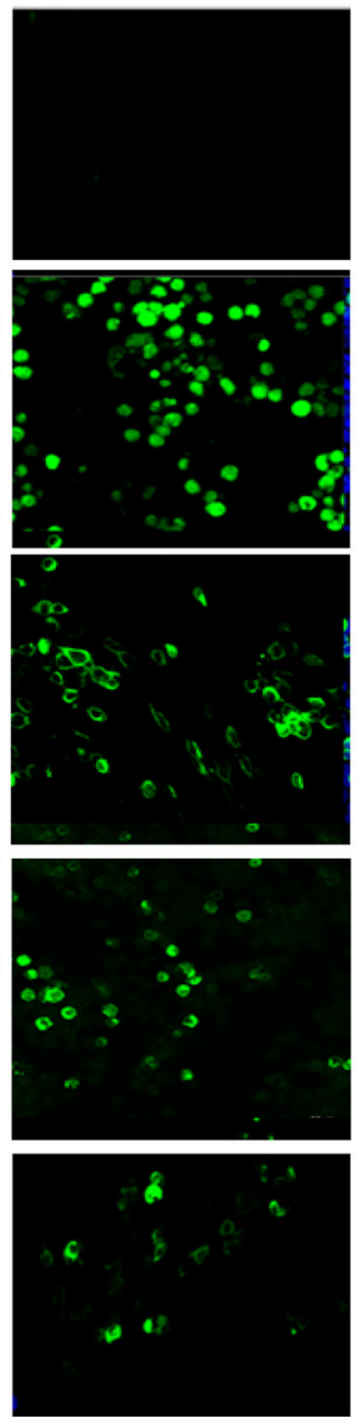

MERGE
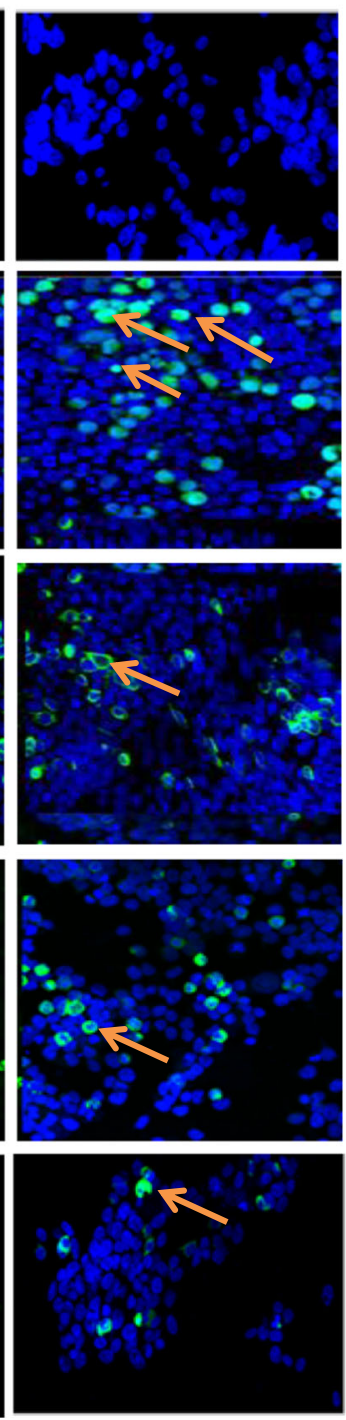
Fig. 3 Growth curves and CEF invasion test. a Growth curves of the 4 strains and $\mathbf{b}$ determination of the invasion rates of the recombinant strains in vitro. By stimulating CEFs and counting the number of intracellular bacteria, the cell invasion rates were calculated. Statistically, $P<0.001$ was statistically significant and marked as triple asterisks $\mathbf{a}$

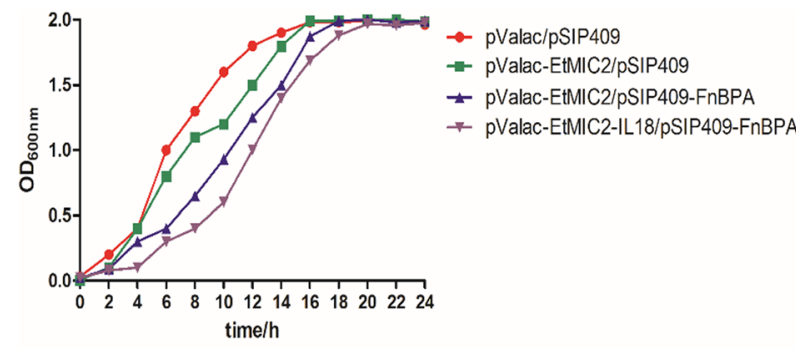

b

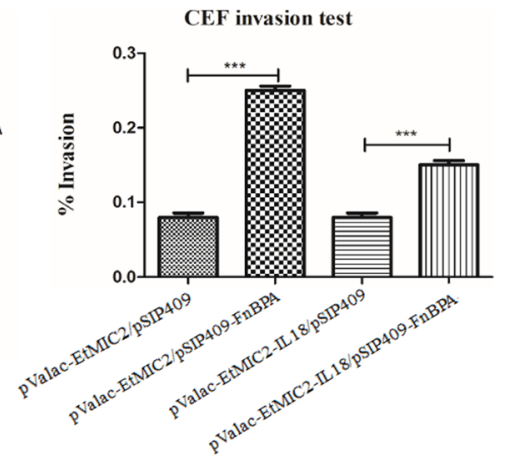

significantly higher than that in group III $(P<0.01)$ (Fig. $4 \mathrm{~b})$. The IL-18 expression levels were significantly different among all groups (Fig. 4d). The results showed that the IL18 protein was successfully expressed in group VI in vivo. Group VI had the highest expression level of IL-4 (Fig. 4c). This result showed that the immunization group including IL-18 adjuvant could induce the host to produce more IFN- $\gamma$ and promote the production of IL-4. The results showed that the SIgA content in
Fig. 4 ELISA test. a The specific antibody EtMIC2 levels of chickens in six groups at 30 days and 38 days. b Detection of IFN$\gamma$ levels in the serum of chickens at 30 days. c Detection of IL-4 levels in the serum of chickens at 30 days. d Detection of IL-18 levels in the serum of chickens at 30 days. e Assay of SIgA in the intestinal lavage fluid of chickens at 30 days. $\mathbf{f}$ Detection results of the FnBPA antibody in the serum at 30 days. $(* P<0.05, * * P<0.01$ and $* * * P<0.001)$
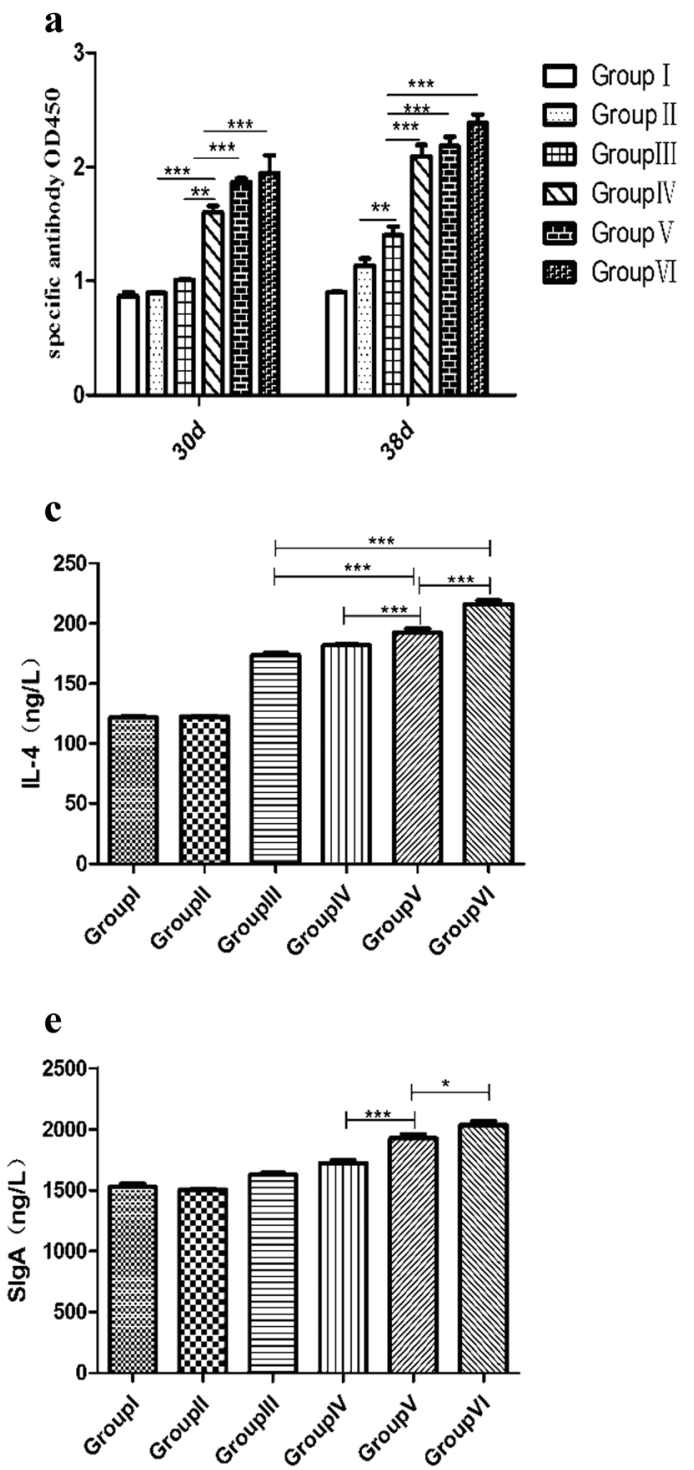

b

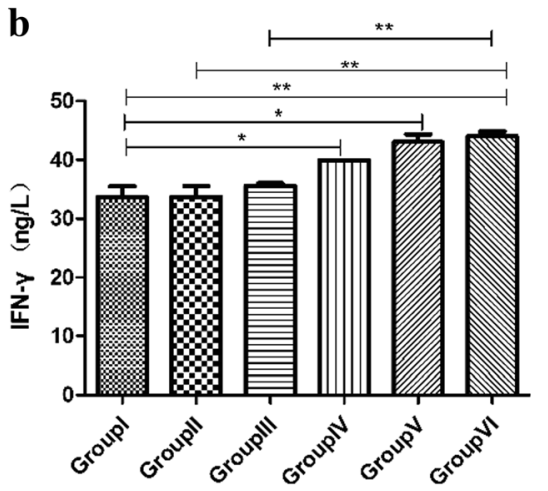

d

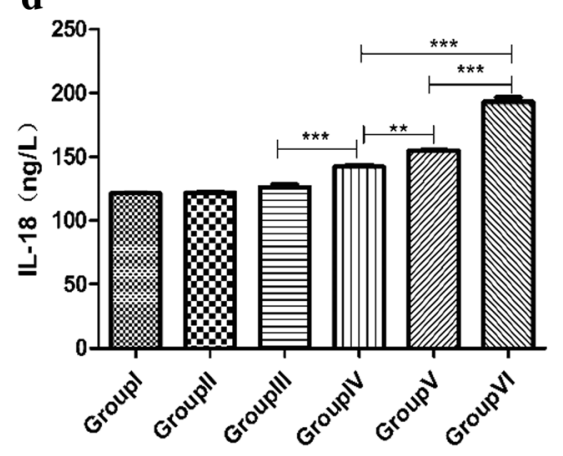

f

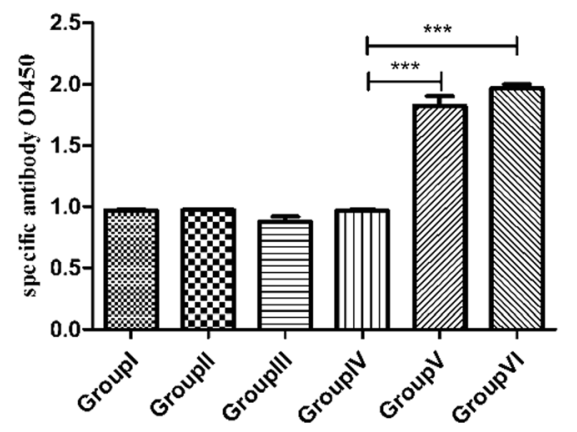


Fig. 5 Lymphocyte proliferation and increased rates of $\mathrm{CD}^{+} \mathrm{CD} 4^{+}$ and $\mathrm{CD}^{+} \mathrm{CD}^{+} \mathrm{T}$ cells. a Single cells were prepared from peripheral blood leukocytes (PBLs) as described, followed by stimulation with concanavalin A $(10 \mu \mathrm{g} / \mathrm{mL})$ for approximately $48 \mathrm{~h}$. Evaluation of cell proliferation status by the MTT assay. b The percentage of CD4+ splenocytes was determined at 30 days and 38 days by FACS. c The percentage of $\mathrm{CD} 8^{+}$ splenocytes was determined at 30 days and 38 days by FACS. $(* P<0.05, * * P<0.01$ and $* * * P<0.001)$ $\mathbf{a}$

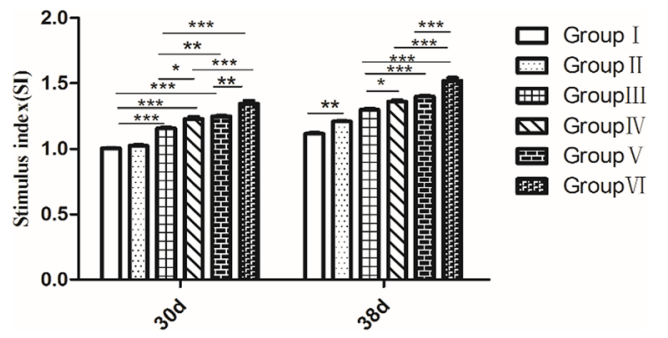

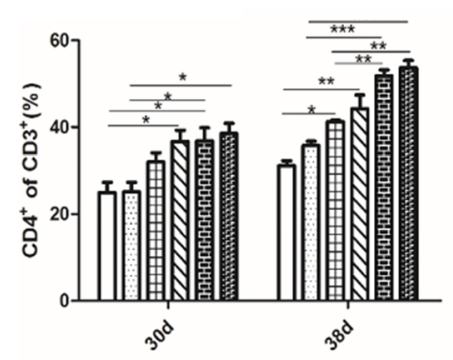
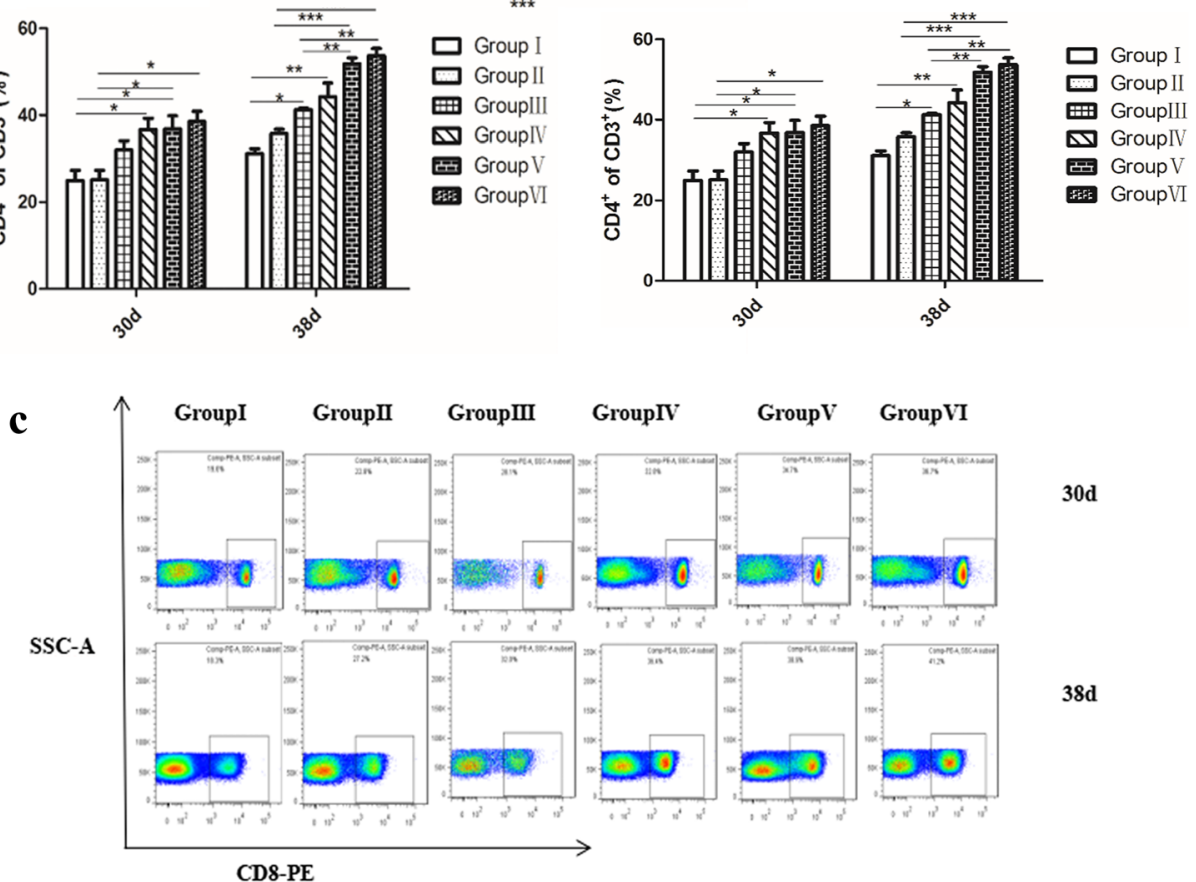

30d

$38 d$
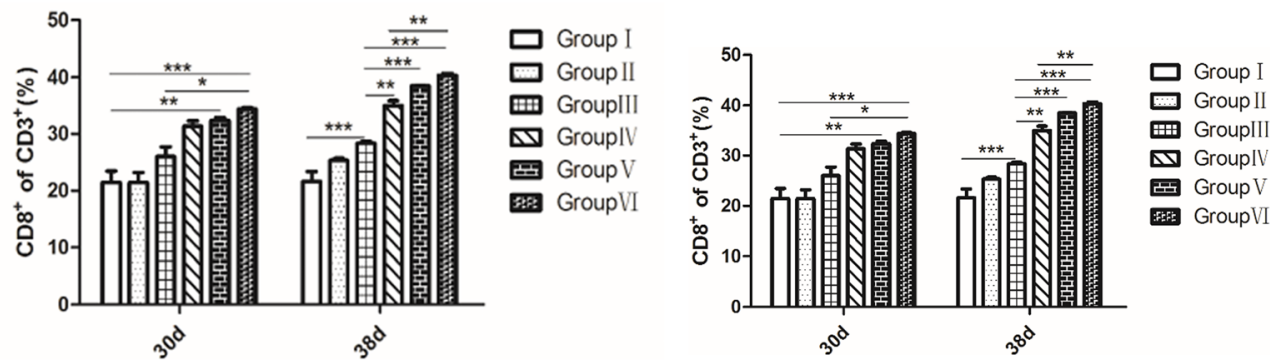
group VI was the highest, which could lead to a stronger mucosal immune response in the chickens (Fig. 4e).

The levels of the FnBPA antibody in the sera of group V and group VI chicks were significantly higher than those in other groups, while there was no significant difference among the other groups. The results showed that the Lactobacillus strain expressing the FnBPA protein in vivo could induce the production of specific antibodies against the FnBPA antigen in the host after entering the chickens (Fig. 4f).

\section{Lymphocyte proliferation and increased rates of $\mathrm{CD}^{+} \mathrm{CD}^{+}$and $\mathrm{CD}^{+} \mathrm{CD}^{+}{ }^{+}$cells}

To assess the effects of immunization with different strains on immune functions, subsets of $\mathrm{CD} 4^{+}$and $\mathrm{CD} 8^{+}$splenocytes and lymphocyte proliferation were evaluated. The lymphocytes from the PBLs were stimulated with concanavalin A (ConA), and the stimulation index (SI) was calculated by ELISA (Fig. 5a). The results showed that group VI had a significantly higher SI than the other groups 8 days after challenge (38 days) $(P<0.001)$, and group VI had a stronger proliferative ability than group $\mathrm{V}(P<0.01)$ before challenge (30 days).

The percentages of $\mathrm{CD}^{+}$and $\mathrm{CD} 8^{+}$splenocytes were determined by FACS. Before challenge, the contents of $\mathrm{CD}^{+} \mathrm{CD}^{+} \mathrm{T}$ lymphocytes in group IV, group $\mathrm{V}$ and group VI were obviously higher than those in normal saline $(P<0.05)$, and there was no significant difference from group III. After challenge, the ratio of $\mathrm{CD}^{+} \mathrm{CD}^{+} \mathrm{T}$ lymphocytes in group VI was the highest (Fig. 5b). In comparison, the contents of $\mathrm{CD}^{+} \mathrm{CD}^{+} \mathrm{T}$ cells in group VI were significantly increased on days 30 and 38 (Fig. 5c). On day 38, the percentages of $\mathrm{CD}^{+} \mathrm{CD}^{+} \mathrm{T}$ cells in chickens of both group $\mathrm{V}$ and group VI were obviously higher than those in chickens of group III $(P<0.001)$ (Fig. 5c). It was proven that the experimental strain enhanced the cellular immunity of the host to some extent.

\section{Protection against parasite challenge}

No chickens died from $E$. tenella challenge in any group. The protective efficacy of $L . P$ against the challenge was evaluated in chickens by examining the relative weight gain rate (RWG), caecal lesions, OPG and ACI. Both the RWG and ACI of group VI were the highest, except for group I (Fig. 6a; Table 3). The group of chickens immunized with pValavEtMIC2-IL18/pSIP409-FnBPA exhibited ACI values greater than 174.75. The ACI value of the pValav-EtMIC2/pSIP409FnBPA group (group V) was slightly lower (approximately 167.66) (Table 3). The OPG levels of group IV, group $\mathrm{V}$ and group VI were significantly lower than those of group III $(P<0.001)$ (Fig. 6c). The LSs of group V and group VI were obviously lower than those of group III $(P<0.05)$ (Fig. 6b). The caecum autopsy (data not shown) and histopathologic analysis showed that the caecum lesions were the most serious in group II. In contrast, group VI had the least serious lesions. Pathological sections of the caecum were observed to determine the extent of lesions in each group. The caecal wall of the group II chicks was obviously thickened, the intestinal villi were broken, the glandular structure was fuzzy and there were a large number of thrombi. The intestinal wall of the group $\mathrm{V}$ and group VI chickens was not significantly thickened, and the intestinal villi were relatively intact, with some inflammatory cells; the degree of damage in the lesions in group $\mathrm{V}$ and group VI was lower than that in the other groups (Fig. 6d). Therefore, all of the results confirmed the protective efficacy of pValac-EtMIC2-IL18/pSIP409-FnBPA.

\section{Discussion}

With the obvious advantages of safety and stability, the DNA vaccine is a new method to prevent and treat coccidiosis, and cytokines are usually used as adjuvants for the DNA vaccine
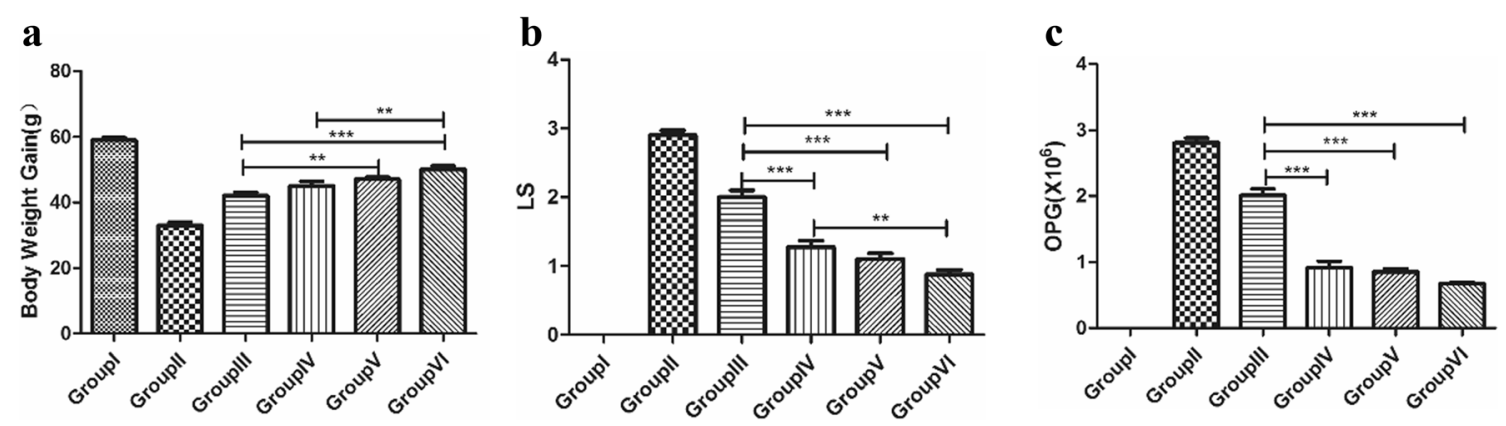

Fig. 6 Protection against E. tenella challenge by all recombinant L.P strains. a The chicken RWG values were measured 8 days postchallenge. b The LS values were recorded until 8 days post-challenge and calculated. $\mathbf{c}$ Statistics of OPG values. $\mathbf{d}$ The caeca were collected and evaluated by histopathologic analysis. $(* P<0.05$, $* * P<0.01$ and $* * * P<0.001$ ) 
d

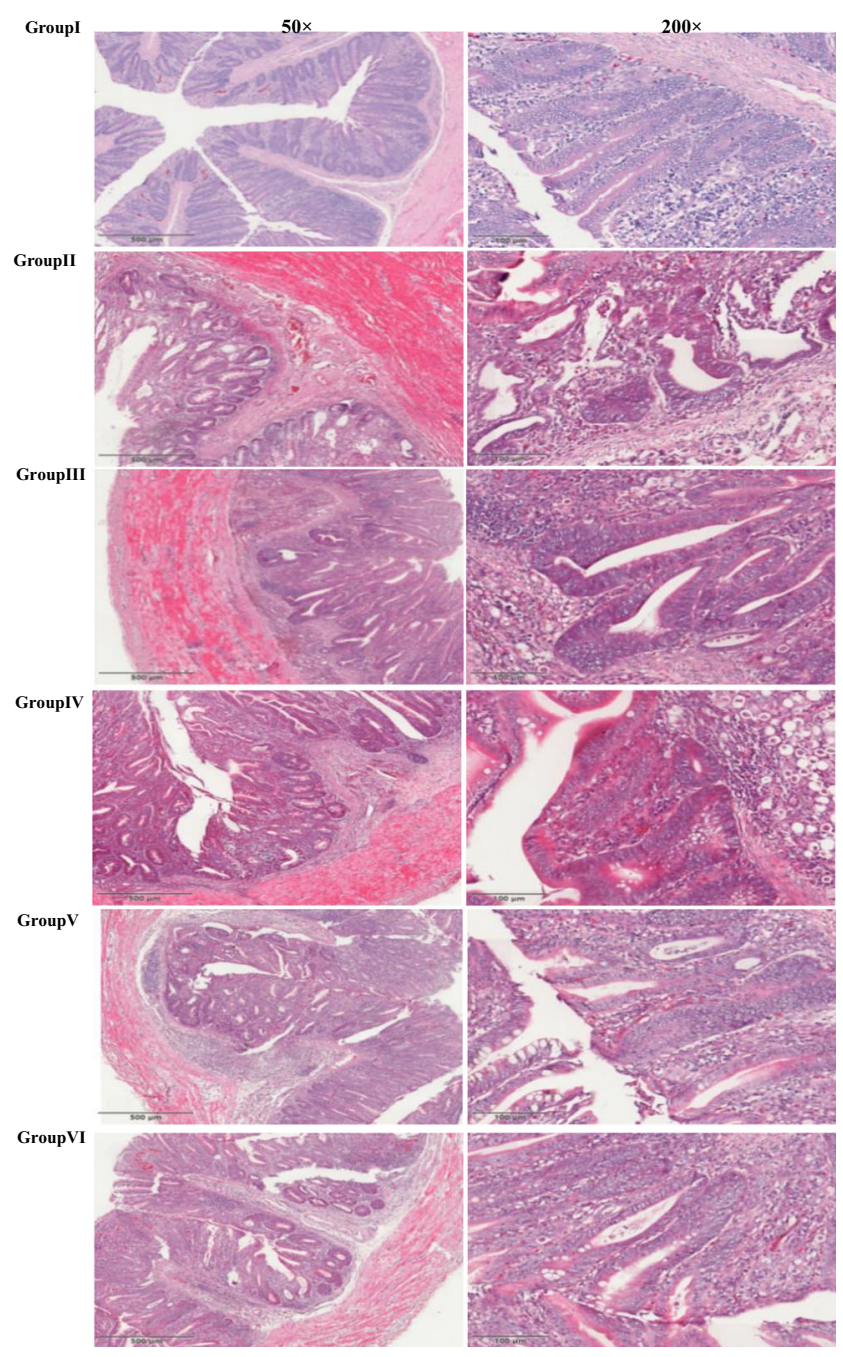

Fig. 6 (continued)

to co-immunize the host and ultimately achieve the goal of enhancing the immune effects of the vaccine.

In recent years, progress has been made in the study of Lactobacillus preparations to protect against coccidiosis. Due to the colonization abilities of LABs, their role in mediating the mucosal immune response and their function in inducing $\mathrm{T}$ and $\mathrm{B}$ lymphocyte activation and cytokine production, LABs provide a new avenue for anti-coccidiosis research. Tierney et al. studied the relationship between Lactobacillus and E. tenella in chickens. By analysing three strains of Lactobacillus isolated from different intestinal segments in chickens, the effect of Lactobacillus on E. tenella invading MDBK cells was observed in vitro. The anticoccidiosis effect produced by the supernatant of the Lactobacillus culture solution indicates that substances secreted by LABs inhibit the invasion of coccidia (Tierney et al. 2004). Therefore, Lactobacillus, an ideal vector for the coccidial DNA vaccine, delivers plasmids to intestinal epithelial cells to fully express exogenous proteins, thus triggering local mucosal immunity and even systemic immunity. Although LABs can be used as living carriers to deliver plasmids expressing foreign proteins to the host, they are not very efficient (Cao et al. 2014). By increasing the invasiveness and adhesion of lactic acid bacteria, bacterial colonization in the intestine is stronger and persists longer, and therefore, foreign proteins can be fully expressed in intestinal epithelial cells. Our experimental results showed that the invading Lactobacillus showed higher invasion of CEFs than did the control strain, and the FnBPA protein could dramatically improve the invasion ability of Lactobacillus.

S. aureus is a pathogenic bacterium that is capable of invading host cells (Sinha and Herrmann 2005). When invading cells, fibronectin (Fn)-binding proteins (FnBPA and FnBPB) bind to the $\alpha 5 \beta 1$ integrin on the cell surface, enabling the bacteria to attach to endothelial cells. Studies have found that Lactococcus lactis (L. lactis) (LL-FnBPA+), which can express fibronectin-binding protein A in S. aureus, has a higher internalization rate in vitro in Caco-2 cells than the original L. lactis strain and can deliver $1 \%$ eukaryotic expression plasmid expressing green fluorescent protein (GFP) to human Caco-2 cells (Pontes et al. 2012). Studies have shown that the use of LL-FnBPA+ can increase the amount of bovine whey protein (BLG) produced by mice, and thus, the invasive strain of Lactobacillus has high potential as a DNA delivery vector in vivo (Pontes et al. 2014). However, the invasion rate of the new invasive anti-coccidiosis L.P constructed in our laboratory has not reached $1 \%$, which may be related to the different shapes (bacilli and cocci) or the plasmids they carry. L. lactis strains expressing FnBPA were able to boost the immune response induced by DNA immunization (Pontes et al. 2014). Another similar result indicated that L. lactis E7 + FnBPA elicited a proinflammatory and mainly systemic immune response that can protect against tumour development when challenged with TC-1 cells; therefore, FnBPA plays an adjuvant role (Almeida et al. 2016). Our experimental results also indicate that FnBPA has a certain adjuvant effect. It also has the ability to invade cells, potentially increasing the efficiency of plasmid delivery.

Although EtMIC2 has been previously expressed by several researchers using HeLa cells, plant cells and E. coli expression systems (Tomley et al. 1996; Jiang et al. 1999; Sathish et al. 2011), it has not been carried via eukaryotic pValac in invasive L.P. Our results confirm that EtMIC2 expressed in chickens had good immunogenicity. Cytokines are involved in inflammatory responses and immune responses, mediating mutual regulation and information exchange between immune cells. IL-2 and INF- $\gamma$ are commonly used adjuvants in coccidial DNA vaccines. Studies have shown the immune effect of pVAX-SO7-IL-2, a microchimeric DNA vaccine of E. tenella, and concluded that IL-2 could be used as an adjuvant to improve the strength of the immune response to the SO7- 
Table 3 Anticoccidiosis index

\begin{tabular}{|c|c|c|c|c|c|}
\hline Groups & $\begin{array}{l}\text { Relative weight } \\
\text { gain rate }(\%)\end{array}$ & $\begin{array}{l}\text { The survival } \\
\text { rate }(\%)\end{array}$ & Oocyst value & $\begin{array}{l}\text { Pathological } \\
\text { change value }\end{array}$ & ACI \\
\hline Group I & 100 & 100 & 0 & 0 & 200 \\
\hline Group II & 55.93 & 100 & 10 & 29 & 116.93 \\
\hline Group III & 71.19 & 100 & 10 & 20 & 141.19 \\
\hline Group IV & 76.27 & 100 & 1 & 13 & 162.27 \\
\hline Group V & 79.66 & 100 & 1 & 11 & 167.66 \\
\hline Group VI & 84.75 & 100 & 1 & 9 & 174.75 \\
\hline
\end{tabular}

antigen DNA vaccine (Song H Y et al. 2013). IL-18, a relatively new adjuvant, can induce Th1 and NK cells to secrete IFN- $\gamma$, promote the differentiation of Th1 cells and increase the killing power of NK cells against coccidians; IFN- $\gamma$ then activates macrophages to secrete more IL-18. Therefore, the use of IL18 as an anti-coccidial DNA vaccine adjuvant has gradually attracted attention (Chen et al. 2011; Li et al. 2013). The plasmids pVAX1-MIC2 and pVAX1-MIC2-IL-18 were developed as DNA vaccines for the inoculation of chicks by intramuscular injection, and the protective effects of the DNA vaccines were then evaluated. The results showed that pVAX1-MIC2-IL-18 exhibited substantially improved immune protection against challenge, which can significantly decrease caecal damage and reduce the oocyst shedding rate after coccidial challenge; the ACI of pVAX1-MIC2-IL-18 reached 171, which was similar to the results in our experiment, and the ACI of pValacEtMIC2-IL18/pSIP409-FnBPA was 174.75. Consistent with the results of this study, the strong immunoadjuvant effect of IL-18 was proven. The ACI of pVAX1-MIC2 was 152, which was obviously lower than that of group V, which reached 164 (Shi et al. 2014). In contrast, we used invasive L.P as a carrier to immunize chickens by convenient oral administration rather than intramuscular injection. Therefore, the invasive $L . P$ strains that we constructed have a good degree of protective efficacy.

Our results are consistent with our expectations. The invasive ability of invasive $L . P$ was significantly higher than that of non-invasive L.P (Fig. 3). Additionally, pValac-EtMIC2IL18/pSIP409-FnBPA had the best anti-coccidiosis effect, indicating that the effect of invasive $L . P$ was better than that of non-invasive L.P. Taken together, the results demonstrated that EtMIC2 was an effective candidate antigen for vaccine development and that chIL-18, as an immune adjuvant, was capable of enhancing the effect of the DNA vaccine. Furthermore, invasive L.P is potentially a novel and efficient DNA delivery vector. However, the role of this invasiveness in enhancing vaccine efficacy will be the focus of further research.

Acknowledgements The pValac-GFP plasmid was kindly provided by Anderson Miyoshi. The plasmid containing FnBPA was provided by
Jean-Marc Chatel (INRA, France). The authors thank Prof. Xin Cao for improving the language in linguistic revision.

Funding information This work was supported by the National Key Research and Development Program of China (2017YFD0501200, 2017YFD0500400, 2017YFD0501000), the National Natural Science Foundation of China $(31672528,31941018)$ and the Science and Technology Development Program of Jilin Province (20180201040NY, 20190301042NY).

\section{Compliance with ethical standards}

All animal husbandry and experimental procedures were performed in accordance with the Chinese Animal Management Ordinance (People's Republic of China Ministry of Health, document No. 55, 2001). The protocol for the animal studies was approved by the Animal Care and Ethics Committees of Jilin Agriculture University.

Conflict of interest The authors declare that they have no conflict of interest.

\section{References}

Almeida JF, Breyner NM, Mahi M, Ahmed B, Benbouziane B, Boas PCBV (2016) Expression of fibronectin binding protein A (FnBPA) from Staphylococcus aureus at the cell surface of Lactococcus lactis improves its immunomodulatory properties when used as protein delivery vector. Vaccine 34:1312-1318

Almeida JF, Mariat D, Azevedo V, Miyoshi A, Leblanc ADMD, Carmen SD (2014) Correlation between fibronectin binding protein A expression level at the surface of recombinant lactococcus lactis and plasmid transfer in vitro and in vivo. BMC Microbiol 14(1):1-8

Cao HP, Wang HN, Zhang AY, Yang X, Ma BC, Xu PW (2014) Research progress of lactic acid bacteria as live vaccine vehicle. China Ani Husb Vet 41(9):258-264

Chen HY, Zhang HY, Li XS, Cui BA, Wang SJ, Geng JW (2011) Interleukin-18-mediated enhancement of the protective effect of an infectious laryngotracheitis virus glycoprotein b plasmid DNA vaccine in chickens. J Med Microbiol 60(1):110-116

Ding X, Lillehoj HS, Dalloul RA, Min W, Sato T, Yasuda A (2005) In ovo vaccination with the Eimeria tenella EtMIC2 gene induces protective immunity against coccidiosis. Vaccine 23(28):3733-3740

Geriletu XL, Xu RH, Li X (2011) Vaccination of chickens with DNA vaccine expressing Eimeria tenella MZ5-7 against coccidiosis. Vet Parasitol 177(1-2):6-12

Gunal M, Yayli G, Kaya O, Karahan N (2006) The Effects of Antibiotic Growth Promoter, Probiotic or Organic Acid Supplementation on 
Performance, Intestinal Microflora and Tissue of Broilers. Int J Poult Sci 5(2):605-609

Helmby H, Jonsson G, Troye-Blomberg M (2000) Cellular changes and apoptosisin the spleens and peripheral blood of mice infected with blood-stage Plasmodium chabaudi chabaudi AS. Infect Immun 68: $1485-1490$

Innocentin S, Guimaraes V, Miyoshi A, Azevedo V, Langella P, Chatel JM (2009) Lactococcus lactis expressing either Staphylococcus aureus fibronectin-binding protein A or Listeria monocyte genes internalin A can efficiently internalize and deliver DNA in human epithelial cells. Appl Environ Microbiol 75:4870-4878

Isolauri E, Sütas Y, Kankaanpãã P, Arvilommi H, Salminen S (2001) Probiotics: effects on immunity. Am J Clin Nutr 73(2Suppl):444s$450 \mathrm{~s}$

Johnson J, Reid WH (1970) Anticoccidial drug: lesion scoring techniques in battery and floor-pen experiment. Exp Parasitol 29:30-36

Jiang J (1999) Expression of microneme gene mic2-7 h in E. coli. Rev Chin Agric Sci Techno 1:022

Kotzamanidis C, Kourelis A, Litopoulou-Tzanetaki E, Tzanetakis N, Yiangou M (2010) Evaluation of adhesion capacity, cell surface traits and immune modulatory activity of presumptive probiotic Lactobacillus strains. Int J Food Microbiol 140(2-3):154-163

Kinoshita M, Miyazaki H, Ono S, Seki S (2013) Immunoenhancing therapy withinterleukin-18 against bacterial infection in immunocompromised hosts after severe surgical stress. J Leukoc Biol 93:689698

Karczewski J, Troost FJ, Konings I, Dekker J, Kleerebezem M, Brummer RJM (2010) Regulation of human epithelial tight junction proteins by Lactobacillus plantarum in vivo and protective effects on the epithelial barrier. Am J Physiol Gastrointest Liver Physiol 298(6): 851-859

Li K, Gao H, Gao L, Qi X, Gao Y, Qin L (2013) Adjuvant effects of interleukin-18 in DNA vaccination against infectious bursal disease virus in chickens. Vaccine 31(14):1799-1805

Liu YL, Li JH, Zhang J, Zhang XC (2011) Construction of an eukaryotic expression vector containing EtMIC-2 gene of Eimeria tenella and its expression in Hela cells. Chinese Vet Sci 31(8):1142-1146

Nolte MA, Hoen EN, van Stijn A, Kraal G, Mebius RE (2015) Isolation of the intact white pulp: quantitative and qualitative analysis of the cellular composition of the splenic compartments. Eur J Immunol 30:626-634

Pontes D, Azevedo M, Innocentin S, Blugeon S, Lefevre F, Azevedo V (2014) Immune response elicited by DNA vaccination using Lactococcus lactis is modified by the production of surface exposed pathogenic protein. PLoS One 9(1):e84509

Pontes DS, Innocentin S, Carmen SD, Almeida JF, Chatel JM (2012) Production of fibronectin binding protein a at the surface of lactococcus lactis increases plasmid transfer in vitro and in vivo. PLoS One 7(9):e44892

Rose ME, Hesketh P (1982) Immunity to coccidia in chickens: adoptive transfer with peripheral blood lymphocytes and spleen cells. Parasite Immunol 4(3):171-185
Sinha B, Herrmann M (2005) Mechanism and consequences of invasion of endothelial cells by Staphylococcus aureus[J]. Thromb Haemost 94(2):266-277

Song X, Huang X, Yan R, Xu L, Li X (2015) Efficacy of chimeric DNA vaccines encoding Eimeria tenella 5401 and chicken IFN- $\gamma$ or IL-2 against coccidiosis in chickens. Exp Parasitol 166(3-4):79-87

Shi WY, Liu Q, Zhang J, Sun J, Jiang X, Geng J (2014) Co-expression of EtMic2 protein and chicken interleukin-18 for DNA vaccine against chicken coccidiosis. Res Vet Sci 97(1):67-70

Song H, Qiu B, Yan R, Xu L, Song X, Li X (2013) The protective efficacy of chimeric SO7/IL-2 DNA vaccine against coccidiosis in chickens. Res Vet Sci 94(3):562-567

Sathish K, Sriraman R, Subramanian BM, Rao NH, Balaji K, Narasu ML (2011) Plant expressed EtMIC2 is an effective immunogen in conferring protection against chicken coccidiosis. Vaccine 29(49): 9201-9208

Shirley MW, Smith AL, Tomley FM (2005) The biology of avian Eimeria with an emphasis on their control by vaccination. Adv Parasitol 60:285-330

Sharman PA, Smith NC, Wallach MG, Katrib M, Meeusen EN, Engwerda CR (2010) Chasing thegolden egg: vaccination against poultry coccidiosis. Parasite Immunol 32(8):590-598

Song X, Zhao X, Xu L, Yan R, Li X (2016) Immune protection duration and efficacy stability of DNA vaccine encoding Eimeria tenella TA4 and chicken IL-2 against coccidiosis. Res Vet Sci 11:31-35

Tomley FM, Bumstead JM, Billington KJ, Dunn PP (1996) Molecular cloning and characterization of a novel acidic microneme protein(Etmic-2) from the apicomplexan protozoan parasite, Eimeria tenella. Mol Biochem Parasitol 79(2):195-206

Tierney J, Gowing H, Sinderen DV (2004) In vitro inhibition of Eimeria tenella invasion by indigenous chicken Lactobacillus species[J]. Vet Parasitol 122(3):171-182

Tomley FM, Soldati DS (2001) Mix and match modules: structure and function of microneme proteins in apicomplexan parasites. Trends Parasitol 17(2):81-88

Vermeulen AN (1998) Progress in recombinant vaccine development against coccidiosis. A review and prospects into the next millennium. Int J Parasitol 28(7):1121-1130

Wong JL, Muthuswamy R, Bartlett DL, Kalinski P (2013) IL-18-based combinatorial adjuvants promote the intranodal production of CCL19 by NK cells and dendritic cells of cancer patients. Oncoimmunology 2(9):e26245

Zhang J, Chen P, Sun H, Liu Q, Wang L, Wang T (2014) Pichia pastoris expressed etmic2 protein as a potential vaccine against chicken coccidiosis. Vet Parasitol 205(1-2):62-69

Publisher's note Springer Nature remains neutral with regard to jurisdictional claims in published maps and institutional affiliations. 